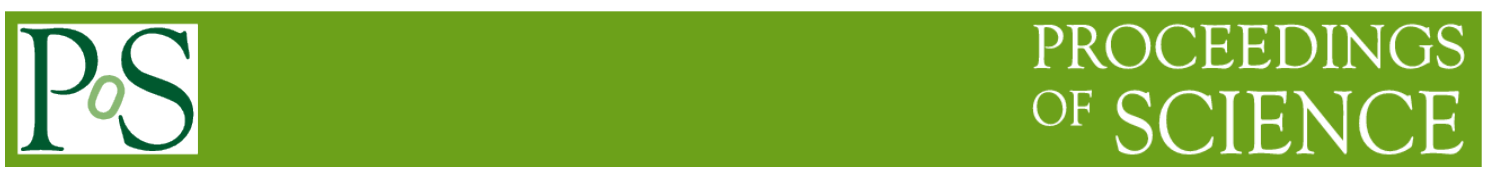

\title{
Age and distance of Puppis A revised - the supernova remnant of the 'Star of Bethlehem'
}

\section{Bernd Aschenbach ${ }^{1}$}

PR Vaterstetten

Mozartstrasse 8, Vaterstetten, Germany

E-mail: bernd.aschenbachat-online.de

Combining the proper motions of the northeast moving oxygen-rich filaments (Winkler et al., 1988 [1]) and the southwest moving neutron star (Becker et al., 2012 [2]) the position of the supernova explosion site and the age of the supernova remnant Puppis A are determined. The filaments are assumed decelerating non-radially but axi-symmetrically. The age is $1990 \pm 150$ yrs. Historical records are checked for a supernova including the 'Star of Bethlehem'. Reviewing the soft X-ray absorption column densities the distance to Puppis A is likely to be $\sim 1.3 \mathrm{kpc}$. The apparent visible magnitude of the Puppis A supernova is estimated to -7 .

XI Multifrequency Behaviour of High Energy Cosmic Sources Workshop 25-30 May 2015

Palermo, Italy

\footnotetext{
${ }^{1}$ Speaker
} 


\section{Introduction}

Puppis A is the name of a supernova remnant (SNR) in the southern sky. It measures $\sim 50^{\prime}$ in diameter. Its distance, based on $\mathrm{HI}$ and $\mathrm{CO}$ measurements, is about $2.2 \mathrm{kpc}$ (Reynoso et al., 2003 [3]), its age has been estimated to be about 4450 yrs (Becker et al., 2012 [2]). The remnant shows radio, infrared, optical and X-ray emission. It is one of the brightest X-ray SNRs in the Galaxy. Based on spectroscopic, mainly optical line measurements, the supernova (SN) has been classified as of type IIb (Chevalier, 2005 [4]). The progenitor was presumably a star with a mass of about 25 solar masses (Canizares \& Winkler, 1981 [5]), which lost a major fraction of its $\mathrm{H} / \mathrm{He}$ envelope before exploding.

Within the remnant oxygen-rich filaments were discovered, quite many of them show high proper motions in the range of 90 to $220 \mathrm{mas} / \mathrm{yr}$. These filaments are all found in the northeast quadrant and are moving towards the northeast boundary of the remnant [1]. The presumably core-collapse SN also left a neutron star (NS) [6], radiating only in X-rays with a pulse period of $112 \mathrm{~ms}$ [7]. The proper motion of the NS was measured and is pointing to the southwest [2], fairly close opposite to the motion of the optical, oxygen-rich filaments. An age estimate of Puppis A was derived by Winkler et al., 1988 [1] using the data of the optical filaments and treating them as some kind of 'bullets' released as such at the time of the explosion and not decelerating since then.

In this paper I combine the measurement data of the oxygen-rich fast-moving filaments and the measurement data of the NS to derive a common explosion site and a common explosion date of the SN. The model for the motion of the filaments involves their non-radial symmetrical but axi-symmetrical deceleration, with deceleration parameters typical for SNRs. The age of Puppis A derived in this way is $\sim 2000$ yrs within a fairly narrow time interval. Among the candidates of unusual celestial phenomena suggested by historical records is the event of 'The Star of Bethlehem'.

Revisiting the information about the distance of Puppis A with emphasis on the low energy $\mathrm{X}$-ray absorption column density a distance of $1.3 \mathrm{kpc}$ is indicated.

\section{The Age}

Figure 1 is a composite of the figure 1 of Winkler et al., 1988 [1] including their figure caption, and an overlay with straight lines, markers and labels, which I added. The original figure of Winkler et al. represents an optical plate which shows eleven fast moving, oxygen-rich filaments (ORFs). The footprints of the arrows mark the position of the ORFs and the length of the arrows indicates the distance travelled for approximately $1000 \mathrm{yrs}$. The ORFs appear to have emerged from a common position, the expansion center (EC), with a positional uncertainty indicated by the ellipse. Arguing that the motion of the ORFs did not decelerate, the time passed since the explosion is $t_{0}=3700 \pm 300$ yrs, i.e., the apparent age of the SNR, according to the analysis of Winkler et al.

Becker et al., 2012 [2] measured the proper motion of the NS to $\mu_{\mathrm{NS}}=71 \pm 12 \mathrm{mas} / \mathrm{yr}$ and the direction of the motion, which, traced back to the past, matches the EC of the ORFs, concluding that the expansion center is the SN explosion site. The position of the NS is off-set from the EC by 6.2 arcmin towards the southwest, which, dividing distance by velocity, results 
in an age of $5200 \pm 1000$ yrs. These two age estimates from the kinematic analysis of the ORFs and the NS, do not overlap within their uncertainty ranges, and it may be worth to consider another model, strengthened by the fact that the center of the radio SNR is also off-set from the EC towards the southwest by 4 arcmin; the NS position and the center of the radio SNR shell are fairly close together, suggesting a closer look at the previous ORF analysis.

I study the case assuming that the ORFs are decelerating with time. I use a self-similarity approach, like it is being done for the expansion of matter driven by a point-like explosion, i.e. the distance $\mathrm{s}$ travelled is proportional to some power of elapsed time $\mathrm{t}$, or $\mathrm{s} \sim \mathrm{t}^{\mathrm{m}}$ with $\mathrm{m}$ the deceleration parameter. Furthermore, I suggest that the deceleration is not radially-symmetric but axi-symmetric, such that $\mathrm{m}$ differs for the two orthogonal directions $\mathrm{x}$ and $\mathrm{y}$, or $\mathrm{s}_{\mathrm{x}} \sim \mathrm{t}^{\mathrm{m}_{\mathrm{x}}}$ and $\mathrm{s}_{\mathrm{y}} \sim \mathrm{t}^{\mathrm{my}}$, with $\mathrm{m}_{\mathrm{x}}$ and $\mathrm{m}_{\mathrm{y}}$ the individual deleceration parameters, respectively. This implies a difference between the currently measured velocity $\mathrm{v}$ and mean velocity $<\mathrm{v}>$, such that $\mathrm{v}_{\mathrm{x}}=\mathrm{m}_{\mathrm{x}}$ $\left\langle\mathrm{v}_{\mathrm{x}}\right\rangle$ and $\mathrm{v}_{\mathrm{y}}=\mathrm{m}_{\mathrm{y}}\left\langle\mathrm{v}_{\mathrm{y}}\right\rangle$. For proper motions $\mathrm{v}$ is to be replaced by the letter $\mu$. The configuration is outlined in figure 1. The EC is marked by ' 3 ', the present position of the NS is marked by ' 5 '. Somewhere between position ' 3 ' and ' 5 ' the $\mathrm{SN}$ went off on the y-axis.

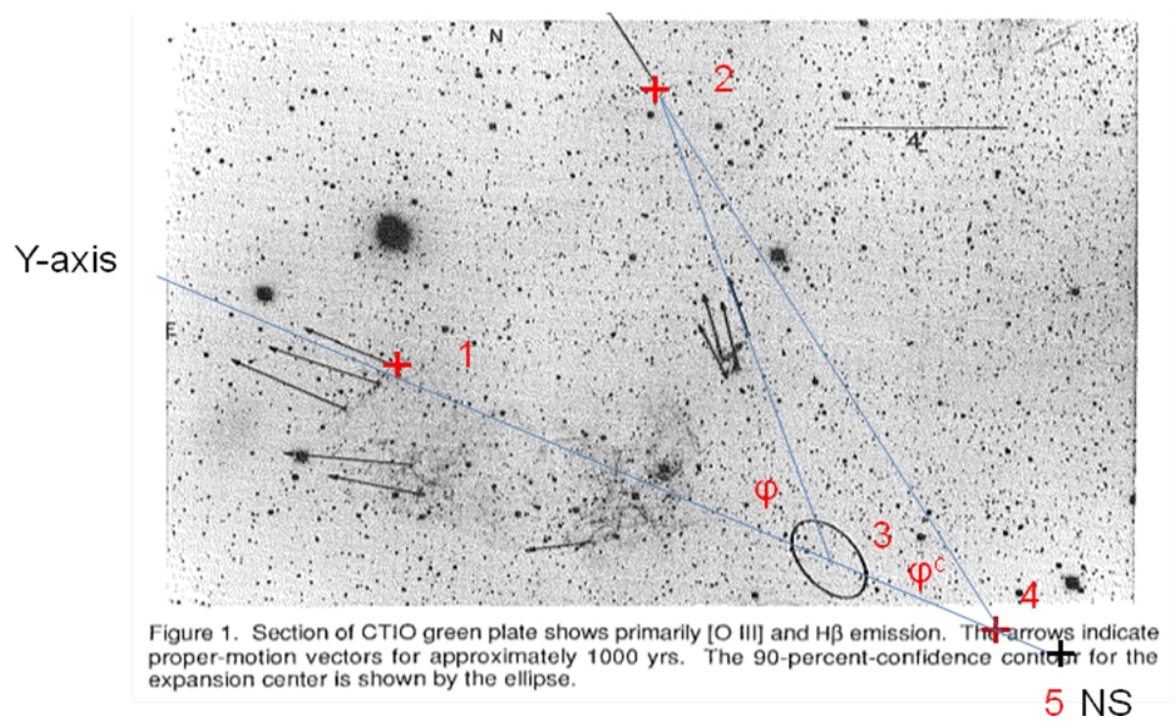

The ORF filament ' 1 ' propagates basically along the y-axis (on-axis), the filament ' 2 ' has the largest off-axis angle $\varphi$, characterizing $\mathrm{x}$ vs. $\mathrm{y}$ deceleration, thereby best suited to discriminate between $m_{x}$ and $m_{y}$.. In the following, distances between various positions are denoted by $S_{i j}$, which are very precisely measured for the positions ' 1 ', '2', '5', except for ' 3 ' with about 1 arcmin uncertainty. Position ' 4 ' would be the 'true' explosion site of the $\mathrm{SN}$, and filament ' 2 ' was 'ejected' under the corrected angle $\varphi^{c}$ from position' 4'. The conditions $S_{14}-S_{13}+S_{45}=S_{35}$ and $\mu_{\mathrm{y}}=\mathrm{S}_{13} / \mathrm{t}_{0}$ lead to equation [1]. With $\mathrm{S}_{35}=6.18^{\prime}, \mathrm{S}_{13}=11.1^{\prime}, \mathrm{t}_{0}=3700$ yrs and $\mathrm{m}_{\mathrm{y}}=0.4, \mathrm{t}=$ $1990 \pm 150 \mathrm{yrs}$, the site of the SN explosion ('4') is located at 2.35' off the present NS position $\left({ }^{\prime} 5^{\prime}\right)$ towards the EC along the y-axis, or RA $(2000)=.08^{\mathrm{h}} 22^{\mathrm{m}} 09^{\mathrm{s}} \operatorname{Dec}(2000)=.-42^{\circ} 59^{\prime} 10^{\prime \prime}$.

$$
\begin{gathered}
\mu_{\mathrm{y}} / \mathrm{m}_{\mathrm{y}} \mathrm{t}-\mu_{\mathrm{y}} \mathrm{t}_{0}+\mu_{\mathrm{NS}} \mathrm{t}=\mathrm{S}_{35} \\
\mathrm{~m}_{\mathrm{y}} \tan (\varphi)=\mathrm{m}_{\mathrm{x}} \tan \left(\varphi^{\mathrm{c}}\right)
\end{gathered}
$$


Maintaining the symmetry with respect to the $\mathrm{y}$-axis the distance along the $\mathrm{x}$-axis needs to be kept. This is equivalent to the expression of equation [2]. Using filament ' 2 ' because of its largest off-axis angle and the relatively lowest proper motion measurement uncertainties, $\mathrm{m}_{\mathrm{x}}=$ 4/7 derived from figure 1. With these estimates of the position of the explosion center, the age, $\mathrm{m}_{\mathrm{x}}$ and $\mathrm{m}_{\mathrm{y}}$ at hand, the position and the proper motion vector of each filament have been checked against the respective measurements delivered by Winkler et. al in their figures 1 and 2 and are found to be consistent within the measurements uncertainties.

The values of the deceleration parameters are close to what is expected from self similarity solutions for the expansion of SNR. The $\mathrm{m}_{\mathrm{y}}=0.4$ represents an adiabatic, Sedov-like expansion in an ambient medium of constant density $(\mathrm{m}=2 / 5)$. The $\mathrm{m}_{\mathrm{x}}$ value could be interpreted as expansion in an ambient medium with a radial density profile declining proportional to $\mathrm{r}^{-3 / 2}$ with $\mathrm{r}$ the distance from the explosion center. Of course, $\mathrm{m}_{\mathrm{y}}=0.4$ has been chosen because of the analogy with the expansion of SNRs. Other choices are possible and higher values of $\mathrm{m}_{\mathrm{y}}$ (lower deceleration) suggest a higher age, but within the uncertainty range of the measurements an age of $\sim 2000$ yrs within a fairly narrow interval is very likely, if some significant decelerating of the ORF's is applicable.

The approach chosen would suggest that the SN exploded in a stratified, close to planeparallel, ambient medium with its normal parallel to the y-axis and the density increasing towards the northeast direction whereas the expansion along the $\mathrm{x}$ - and $\mathrm{z}$-directions are in an evolutionary phase between propagation in the progenitor's stellar wind and the Sedov asymptotic.

Some evidence for this configuration might be obtained from figure 2, which shows the Xray image of Puppis A published by Dubner et al., 2013 [8].

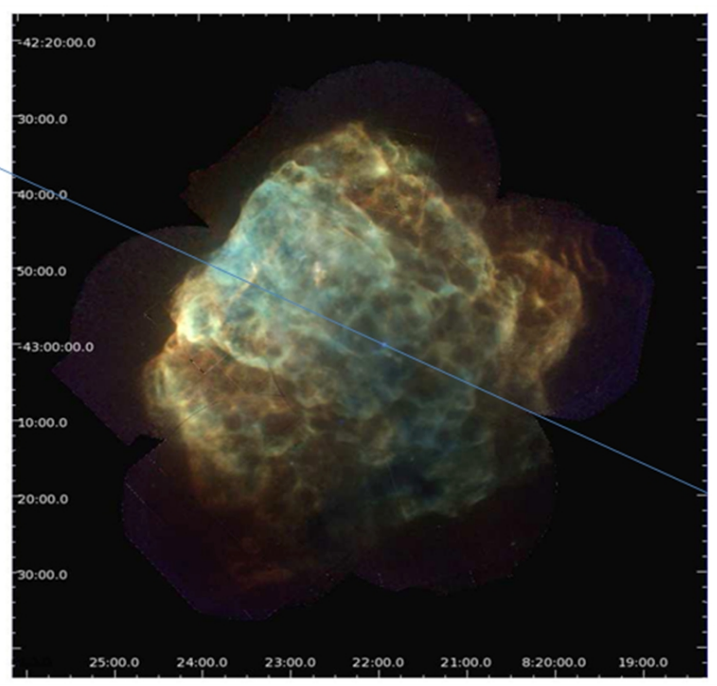

Figure 2: (RA, Dec) ${ }_{2000}$ X-ray brightness image of Puppis A (Dubner et al., 2013 [8]).

The diagonal line delineates the y-axis shown in Fig. 1.

The straight line marks the y-axis, i.e., the 'explosion axis'. It runs through the NS position and separates the remnant axi-symetrically in two parts and is almost parallel to the normal of the fairly straight northeast boundary of the remnant, towards which also the brightness increases, which in turn increases with the ambient density squared. The normal and the almost straight 
delineation of the remnant's northeast boundary might get some meaning when viewing the remnant in galactic coordinates, which is shown in figure 3.

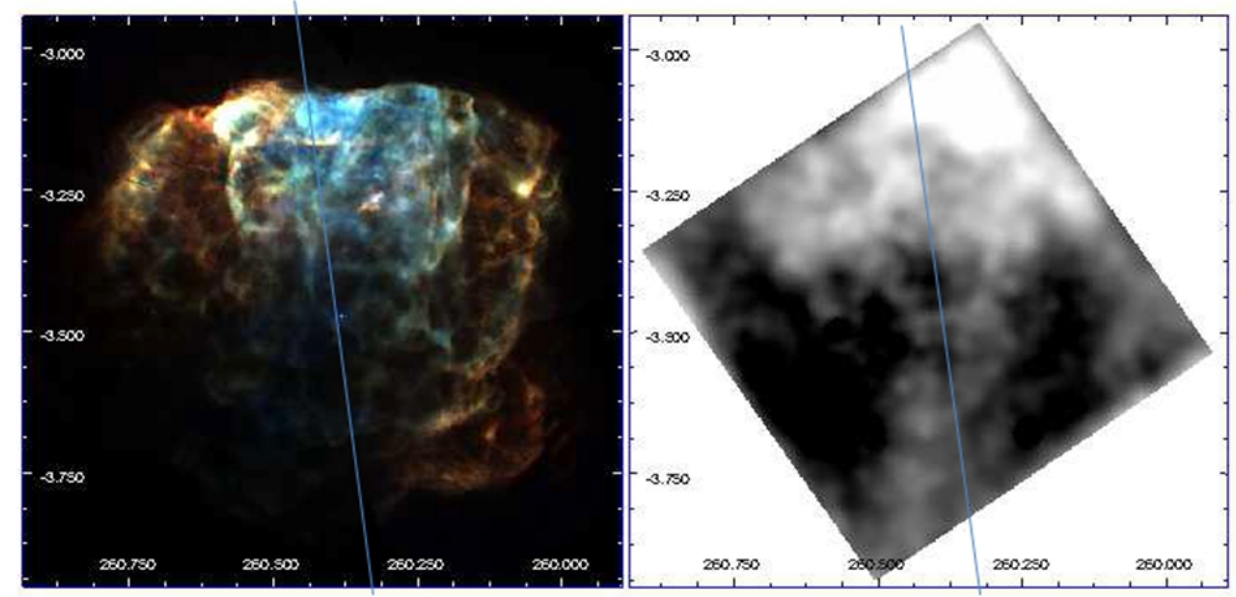

Figure 3a (left): Same as Fig. 2 in galactic coordinates, galactic plane towards the top.

Figure 3b (right): Neutral hydrogen column density $(\mathrm{NH})$ integrated up to the systemic velocity of Puppis A. Note the lower NH away from the galactic plane (towards the bottom) and outside the vertical lane (Dubner et al., 2013 [8]).

Figure 3a demonstrates that the 'explosion axis' is approximately parallel to the normal of the galactic plane. Figure $3 \mathrm{~b}$ shows the distribution of the neutral hydrogen $(\mathrm{NH})$ integrated up to the systemic velocity of Puppis A [8]. Two components are visible. $\mathrm{NH}$ values increase towards the galactic plane and drop along the direction below the NS position (galactic south). The second NH component is the vertical band which coincides with the blue lane of figure $3 \mathrm{a}$, even beyond the galactic southern boundary of Puppis A.

\section{The Distance}

A distance to Puppis A of $2.2 \mathrm{kpc}$ has been derived from studies of $\mathrm{HI}$ and $\mathrm{CO}$ distributions [3]. In contrast, the study of $\mathrm{OH}$ molecules suggests a distance of $1.3 \mathrm{kpc}$ (Woermann et al., 2000 [9]). Spatially resolved X-ray spectra across Puppis A show a systematic variation of the X-ray absorbing column density $\mathrm{N}_{\mathrm{H}}$ across the remnant with the highest column density in the blue lane of figure $3 \mathrm{a}$, which coincides with the lane of $\mathrm{NH}$ excess, figure $3 \mathrm{~b}$. I suggested that this lane, possibly a foreground effect, causes extra absorption (Aschenbach, 1993 [10]). Measurements of the X-ray absorption outside this lane, left- and right-ways, show a significantly lower column density and are therefore more representative as a distance indicator.

Puppis A, projected on the sky, is very close to the Vela SNR, as shown in figure 4a. At its northwest corner Puppis A shows up. The colours represent the hardness of the X-ray spectrum, with blue being the hardest. As can be seen there is an even harder spectrum for a lane stretching across the Puppis A remnant (dark blue). It is coincident with the neutral hydrogen $\mathrm{NH}$ lane shown in figure $3 \mathrm{~b}$; it is a region of higher X-ray absorption with a total $\mathrm{N}_{\mathrm{H}}=3.3 \cdot 10^{21}$ $\mathrm{cm}^{-2}([10,11])$. Outside of this lane the X-ray absorption column density $\mathrm{N}_{\mathrm{H}}=1.6 \cdot 10^{21} \mathrm{~cm}^{-2}$ (Aschenbach, 1993 [10]), later confirmed by Hwang et al., 2008 [11]) with $\mathrm{N}_{\mathrm{H}}=1.5 \cdot 10^{21} \mathrm{~cm}^{-2}$. 
In the following I assume that the latter $\mathrm{N}_{\mathrm{H}}$ value outside the blue lane is a representative measure for the distance.

Lu \& Aschenbach, 2000 [12] have mapped the $\mathrm{N}_{\mathrm{H}}$ distribution across the Vela SNR, which is shown in figure $4 \mathrm{~b}$. In the area closest to Puppis A at the outermost edge of the Vela SNR $\mathrm{N}_{\mathrm{H}, \text { vela }}=3 \cdot 10^{20} \mathrm{~cm}^{-2}$. Scaling with the distance to the Vela SNR, which is fairly well known, i.e. $250 \mathrm{pc}$ (Cha et al., 1999 [13]) and $\mathrm{N}_{\mathrm{H}, \text { Puppis }}=1.5 \cdot 10^{21} \mathrm{~cm}^{-2}$, the distance to Puppis A is $1.3 \mathrm{kpc}$, which is in agreement with the $\mathrm{OH}$ measurements.

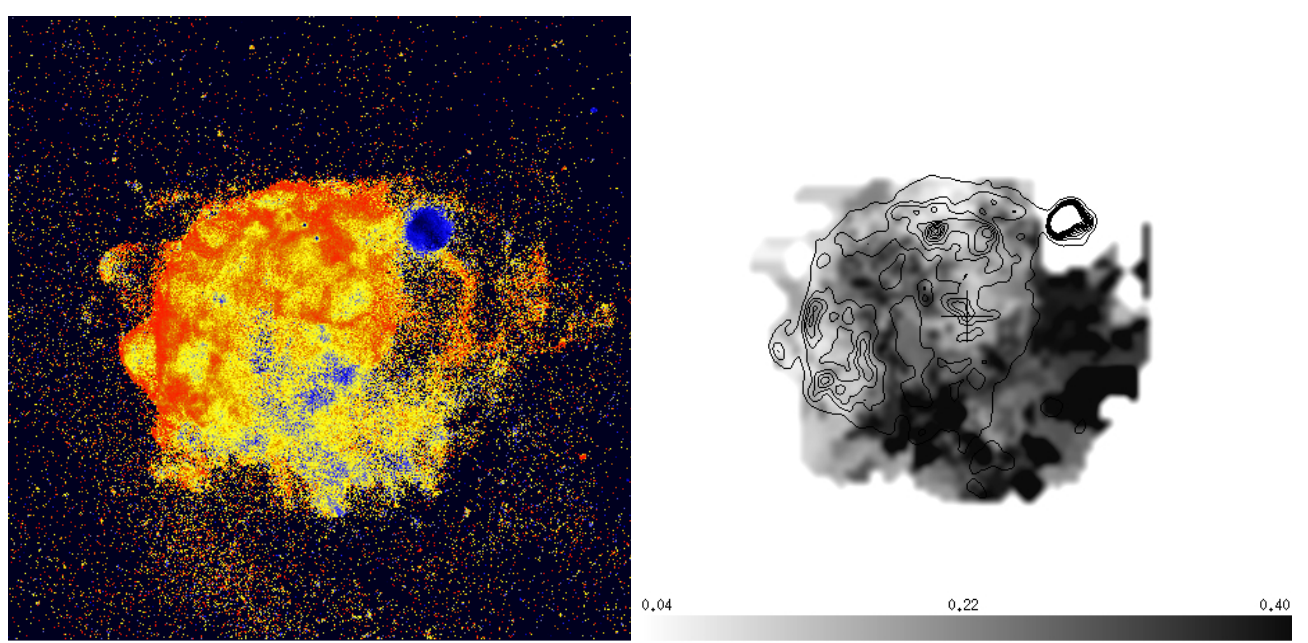

Figure 4a (left): X-ray colour image of the Vela SNR including Puppis A at the upper right corner (dark blue) with the almost black lane crossing.

Figure 4b (right): X-ray absorbing column density $\mathrm{N}_{\mathrm{H}}$, contour lines and grey scale, of the Vela SNR with Puppis A as black-encircled white patch at the upper right. Grey scale at the bottom is in units of $10^{21} \mathrm{~cm}^{-2}$ (Lu \& Aschenbach, 2000 [12]).

As a side effect, with $d=1.3 \mathrm{kpc}$, the space velocity of the Puppis A NS is $440 \pm 75 \mathrm{~km} / \mathrm{s}$, which is still high, but does not invoke a hypervelocity object.

\section{The Supernova}

Based on the distance of $1.3 \mathrm{kpc}$ and using the light curve of the SN prototype IIb, SN1993J in M81, apparent visual magnitudes $\mathrm{m}_{\mathrm{v}}$ for the Puppis A SN are estimated as follows; peak: -7.2 , first minimum at day 5: -4.9 , secondary maximum at day 17: -6.6 , at four months: -4.6 , at one year: -2.2 , at 2.5 years: 2.8 , at 4 years: 5 , after which the star started to get unobservable with the unaided eye. With the progenitor stars identified it turns out that SN1993J and SN1987A as well brightened in the visible band by $\sim 10$ magnitudes compared to their progenitors. Thus the progenitor of the Puppis A SN would have been as bright as $\mathrm{m}_{\mathrm{v}} \sim 2.8$, and it may have been well known to the contemporaries and before, and the SN might have been regarded as some flaring of a known star rather than a new star.

Interestingly, the lids of wooden sarcophagi of Pharaohs of ancient Egypt are decorated with inscriptions which represent star catalogues. They were the basis for attempts to construct a 
calendar, in particular the so-called 'Ramesside decanal clocks', which are suggested to have been created around 1450 BC. Leitz, 1995 [14] has published a identification of each of the stars which appeared to been used for this purpose. In the context of this work one identification is interesting, it is the star q Puppis with a magnitude $\mathrm{m}_{\mathrm{v}}=4.44$, which is fairly faint to have served as a calendar 'guide' star, stressed by the fact that is missing in Ptolemy's Almagest. Instead, I propose the progenitor of the Puppis A SN to be the star in question because of its significantly higher brightness of estimated $\mathrm{m}_{\mathrm{v}} \sim 2.8$. It is located at almost the same right ascension with a difference of $\sim 15 \mathrm{~min}$, and a position $6.5^{\circ}$ further south the latter of which makes basically no difference as far as the timing of the star's rising and setting is concerned .

For the epoch $1990 \pm 150$ yrs back in time, i.e., $\sim 150 \mathrm{BC}$ to $\sim 150 \mathrm{AD}$, Chinese historical records show some time-relevant entries but their celestial coordinates do not have any SNR counterpart within $<20^{\circ}$, which is significantly larger than the typical angular uncertainties of the Chinese record data with respect to events having been identified as SN. An interesting case however is the event of $107 \mathrm{AD}$ which is the most southerly event reported. The position is not well known and may be imprecise by more than one hour in right ascension, and a couple of degrees further south would bring it close to Puppis A. Further study of the historical records may clear the situation.

Another candidate for a SN in this time period is 'The Star of Bethlehem', which is known from the gospel of Matthew 2, 1-9, in the Bible's New Testament. According to historians the time of the 'The Star'appearance may be 7-4 BC or later up to about 110 AD. This is compatible with the age derived in this work for Puppis A. There is to my knowledge no information about the constellation within which 'The Star' appeared. The Puppis A SN lightning up deep in the southern sky could have been visible from geographical latitudes like that of Bethlehem from October to May.

There have been many prophesies, myths and legends concerning 'The Star', which I have no right to comment. This paper simply suggests a SN/SNR candidate for 'The Star'. As far as the myths are concerned, I cannot resist mentioning a curious observation. A straight line from Sirius, the brightest star in the sky, to the center of Puppis A, passes at half of their angular separation through another bright star in Canis Majoris with the name Aludra $\left(\mathrm{m}_{\mathrm{v}}=2.4\right)$, which is Arabic and means 'Virgin' or 'Virginity'.

At last, the SNR RX J0852-4622 (Aschenbach, 2013 [15]) might be another supernova remnant candidate for 'The Star of Bethlehem'. The kinematic age of the SNR has been estimated to be between 1200 and 2200 yrs (Katsuda et al., 2008 [16]), if the SNR is in its adiabatic evolutionary phase. That time span expands to 700 til 4300 yrs if a different evolutionary phase were applied. Allen et al., 2015 [17] applied the same analysis method but at a different rim location of the SNR and conclude that the SNR is between 2.4 and $5.1 \mathrm{kyr}$ old. Early estimates of Aschenbach et al., 1999 [18] suggest an age between 600 and 800 yrs.

\section{References}

[1] P. F. Winkler, J. H. Tuttle, R. P. Kirshner, M. J. Irwin 1988, Kinematics of oxygen-rich filaments in Puppis A, in proceedings of IAU Colloq. 101, Supernova Remnants and the Interstellar Medium', eds. R. S. Roger \& T. L. Landecker, 65.

[2] W. Becker, T. Prinz, P. F. Winkler, R. Petre 2012, Proper Motion of the Central Compact Object RX J0822-4300 in the Supernova Remnant Puppis A, ApJ 755, 141. 
[3] E. M. Reynoso, A. J. Green, S. Johnston, et al. 2003, Observations of the neutral hydrogen surrounding the radio-quiet neutron star RX J0822-4300 in Puppis A, MNRAS 345, 671.

[4] R. A. Chevalier 2005, Young Core-Collapse Supernova Remnants and Their Supernovae, ApJ 619 , 839.

[5] C. R. Canizares, P. F. Winkler 1981, Evidence for elemental enrichment of Puppis A by a Type II supernova, ApJ 246, 33.

[6] R. Petre, C. M. Becker, P. F. Winkler 1996, A Central Stellar Remnant in Puppis A, ApJ 465, L43.

[7] E. V. Gotthelf, J. P. Halpern 2009, Discovery of a $112 \mathrm{~ms}$ X-Ray Pulsar in Puppis A: Further Evidence of Neutron Stars Weakly Magnetized at Birth, ApJ 695, L35.

[8] G. Dubner, N. Loiseau, P. Rodriguez-Pascual, et al. 2013, The most complete and detailed X-ray view of the SNR Puppis A, A\&A 555, 9.

[9] B. Woermann, M. J. Gaylard, R. Otrupcek 2000, Hydroxyl lines towards Puppis A, MNRAS 317, 421.

[10] B. Aschenbach 1993, X-ray emission from supernova remnants observed with ROSAT, AdSpR 13, 45.

[11] U. Hwang, R. Petre, K. A. Flanagan 2008, X-Ray-emitting Ejecta in Puppis A Observed with Suzaku, ApJ 676, 378.

[12] F. Lu, B. Aschenbach 2000, Spatially resolved X-ray spectroscopy of the Vela supernova remnant, A\&A 362, 1083.

[13] A. N. Cha, K. R. Sembach, A. C. Danks 1999, The Distance to the VELA Supernova Remnant, ApJ 515, L25.

[14] C. Leitz 1995, 'Altägyptische Sternuhren', Orientalia Lovaniensia Alalecta, Peters Publisher, Belgium, ISN 90-6831-669-9.

[15] B. Aschenbach 1998, Discovery of a young nearby supernova remnant, Nature 396, 141.

[16] S. Katsuda, H. Tsunemi, K. Mori 2008, The Slow X-Ray Expansion of the Northwestern Rim of the Supernova Remnant RX J0852.0-4622, ApJ 278, L35.

[17] G. E. Allen, K. Chow, T. DeLaney et al. 2015, On the Expansion Rate, Age, and Distance of the Supernova Remnant G266.2-1.2 (Vela Jr.), ApJ 798, 12.

[18] B. Aschenbach, A. Iyudin, V. Schönfelder 1999, Constraints of age, distance and progenitor of the supernova remnant $R X$ J0852.0-4622/GRO J0852-4642, A\&A 350, 997. 